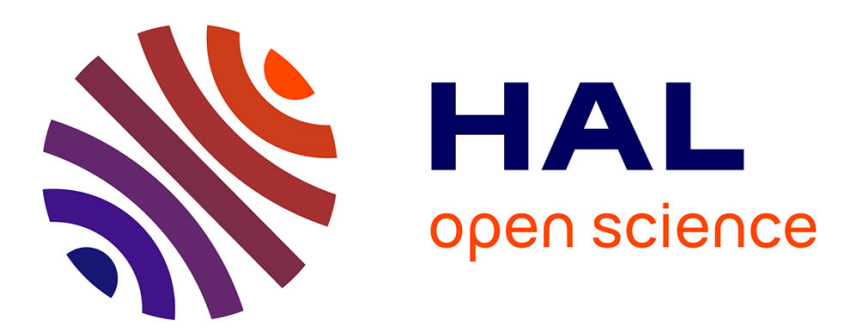

\title{
Les derniers progrès du calibrage des âges radiocarbone permettent-ils une révision des chronologies entre 25 et 50.000 ans B.P.?
}

\author{
Michel Fontugne
}

\section{To cite this version:}

Michel Fontugne. Les derniers progrès du calibrage des âges radiocarbone permettent-ils une révision des chronologies entre 25 et 50.000 ans B.P.?. Quaternaire, 2004, 15 (3), pp.245-252. 10.3406/quate.2004.1771. hal-02470846

\section{HAL Id: hal-02470846 \\ https://hal.science/hal-02470846}

Submitted on 19 Oct 2020

HAL is a multi-disciplinary open access archive for the deposit and dissemination of scientific research documents, whether they are published or not. The documents may come from teaching and research institutions in France or abroad, or from public or private research centers.
L'archive ouverte pluridisciplinaire HAL, est destinée au dépôt et à la diffusion de documents scientifiques de niveau recherche, publiés ou non, émanant des établissements d'enseignement et de recherche français ou étrangers, des laboratoires publics ou privés. 


\title{
LES DERNIERS PROGRÈS DU CALIBRAGE DES ÂGES RADIOCARBONE PERMETTENT-ILS UNE RÉVISION DES CHRONOLOGIES ENTRE 25 ET 50.000 ANS B.P.?
}

\author{
Michel FONTUGNE*
}

\section{RÉSUMÉ}

En énonçant, en 1946, les principes de la méthode de datation par le carbone 14, W.F. Libby réaffirmait le concept de datation absolue et révolutionnait les sciences du Quaternaire. Au fil des ans, à mesure que les performances analytiques et les connaissances en géochimie et biologie s'accroissaient, les limitations de cette méthode sont appanues : les âges ${ }^{14} \mathrm{C}$ étaient notoirement trop jeunes. La principale raison de cette dérive des âges radiocarbone est la variation de la concentration en ${ }^{14} \mathrm{C}$ de l'atmosphère qui dépend à la fois de la production de cet isotope dans la haute atmosphère et des échanges entre les différents réservoirs de carbone sur Terre. La méthode reste néanmoins applicable si l'on peut connaitre les variations de la teneur en ${ }^{14} \mathrm{C}$ atmosphérique dans le passé ; c'est tout le principe du calibrage des âges radiocarbone. Différentes techniques ont été utilisées pour produire ces courbes de calibrage qui se sont révélées contradictoires pour la période entre $25-45 \mathrm{ka}$. Un état actuel de la question est présenté.

Mots-clés : Carbone 14, calibrage.

\section{ABSTRACT}

DO THE LAST PROGRESS OF THE CALIBRATION OF THE RADIOCARBON AGES ALLOW A REVISION OF CHRONOLOGIES BETWEEN 25 AND 50,000 YEARS B.P.?

By stating, in 1946, the principles of the carbon-14 dating method, W.F. Libby reaffirmed the concept of absolute dating and revolutionised Quaternary sciences. With the passing of years, as the analytical performances and knowledge in geochemistry and biology increased, the limitations of this method appeared: the ages ${ }^{14} \mathrm{C}$ were manifestly too young. The principal reason of this drift of the radiocarbon ages is the variation of the concentration in ${ }^{14} \mathrm{C}$ in the atmosphere which depends both on the production of this isotope in the upper atmosphere and on its exchanges between the various carbon tanks on Earth. The method remains nevertheless applicable if one can know the variations of the atmospheric content of ${ }^{14} \mathrm{C}$ in the past; it is the principle of the calibration of the radiocarbon ages. Various techniques were used to produce these curves of calibrations, which appeared contradictory for the period between $25-45 \mathrm{ka}$. A current state of the question is presented.

Key-words: Carbon 14, calibration.

\section{INTRODUCTION}

Depuis une cinquantaine d'années, les Quaternaristes utilisent la méthode de datation par le carbone 14 pour reconstituer et comprendre l'histoire de l'Homme et de son environnement au cours des 50.000 dernières années. Cette technique considérée d'emblée comme une méthode de datation absolue allait très vite être remise en cause. Les âges obtenus notamment pour des échantillons historiques se révélaient trop jeunes, $d$ 'autant plus rajeunis qu'ils étaient anciens. Des premières corrections ont commencé à être appliquées notamment en utilisant une valeur de la période de désintégration radioactive du ${ }^{14} \mathrm{C}$ plus exacte que la période de Libby ou période conventionnelle ( $5568 \pm 30$ ans) qui sous-estimait les âges de $3 \%$. Plus tard les corrections de fractionnement biologique (correction de $\delta^{13} \mathrm{C}$ ) furent introduites (Stuiver et Pollach, 1977). Néanmoins, toutes ces corrections n'expliquaient pas la "dérive" du carbone 14.

La méthode de datation par le carbone 14, dans son principe est simple. Le carbone 14 est produit principalement par l'action des protons cosmiques sur les noyaux des atomes dans la haute atmosphère. Ce carbone 14 se distribue de manière quasi-uniforme dans les basses

* Laboratoire des Sciences du Climat et de l'Environnement, UMRI572. CEA/CNRS, Avenue de la Terrasse, F-9II98-GIF-SUR-YVETTE cedex. e-mail : Michel.Fonhugne(alsce.cnrs-gifffr 
couches de l'atmosphère et dans le monde vivant (voir Délibrias, 1985 ; Evin, 2002). Ainsi, si l'on connaît la quantité de carbone 14 contenue dans un fossile de son vivant, la seule mesure de la quantité de carbone 14 résiduel permet de calculer, grâce à la loi de désintégration radioactive, le temps qui s'est écoulé depuis la mort du fossile. Comme l'activité initiale de carbone 14 des fossiles datés n'était pas connue, Libby proposa qu'elle soit constante dans le temps et égale à l'activité du carbone atmosphérique de l'année 1950 (d'où l'origine des âges ${ }^{14} \mathrm{C}$ B.P., Before Present). Grâce aux premiers travaux de $H$. De Vries à la fin des années cinquante, il est apparu que l'hypothèse simplificatrice de Libby concernant l'invariabilité de la teneur en ${ }^{14} \mathrm{C}$ atmosphérique, n'est pas rigoureusement vérifiée. Il fallait donc pour pouvoir utiliser cette méthode de datation être capable de reconstituer les variations de la teneur en ${ }^{14} \mathrm{C}$ de l'atmosphère dans le passé. Ainsi de ce constat, est née l'idée du calibrage (ou calibration pour les anglo-saxons) des âges ${ }^{14} \mathrm{C}$. Les faits étant bien établis, il restait aux géo-chronologues à comprendre pourquoi cette dérive des âges ${ }^{14} \mathrm{C}$ existait et à mettre en place les moyens de les corriger. Après quarante ans d'efforts et de notoires progrès, les problèmes sont loin d'être résolus dans leur totalité. Il est donc utile, aujourd'hui, de faire le point et de délimiter le champ d'application de la méthode en terme de datation absolue. C'est le but de cet article.

\section{POURQUOI FAUT-IL CORRIGER LES DATATIONS CARBONE 14 ?}

Dans la plupart des méthodes de datation basées sur la décroissance radioactive, on procède à la fois à la mesure de l'isotope " père " $\left({ }^{14} \mathrm{C}\right)$ et de l'isotope " fils" $\left({ }^{14} \mathrm{~N}\right)$ produit par désintégration. Pour des raisons assez évidentes liées à la nature organique des échantillons datés et à l'imprégnation de tous les matériaux par l'azote 14 atmosphérique ambiant, il n'est pas possible de procéder ainsi : seul le carbone 14 résiduel peut être mesuré. Cette difficulté technique avait conduit Willard Libby à contourner le problème en supposant la teneur en ${ }^{14} \mathrm{C}$ de l'atmosphère constante dans l'atmosphère et égale à celle de l'année 1950 (année de référence B.P.). L'hypothèse était crédible cependant, certains, dès la fin années cinquante, pressentaient les faiblesses de cette approche sans pour autant en estimer les conséquences réelles.

En réalité, compte tenu de son mode de production et de son incorporation dans le cycle du carbone global, la teneur en ${ }^{14} \mathrm{C}$ n'a pas été constante (fig. 1). Trois paramètres contrôlent la teneur en ${ }^{14} \mathrm{C}$ de l'atmosphère : le flux de particules cosmiques incidentes, l'intensité du champ magnétique terrestre qui contrôlent la production $\mathrm{de}^{14} \mathrm{C}$ dans la haute atmosphère et les échanges et/ou la répartition entre les différents réservoirs de carbone que sont l'océan, la biosphère et l'atmosphère.

\section{- LA PRODUCTION}

Les variations rapides du rapport ${ }^{14} \mathrm{C} /{ }^{12} \mathrm{C}$ sont liées à des fluctuations magnétiques du Soleil qui modulent l'arrivée sur Terre des protons du rayonnement cosmique. De nombreux travaux, dont ceux de Stuiver et Quay (1980) et de Damon et Linick (1986), montrent que l'activité ${ }^{14} \mathrm{C}$ crôt ou décroît avec les divers minima ou maxima d'activité solaire pour les derniers millénaires. Ce phénomène rend compte des petites oscillations (wiggles) de haute fréquence entraînant une fluctuation des teneurs en ${ }^{14} \mathrm{C}$ de l'ordre de $\pm 5 \%$. Par contre, la décroissance de 30 à $50 \%$

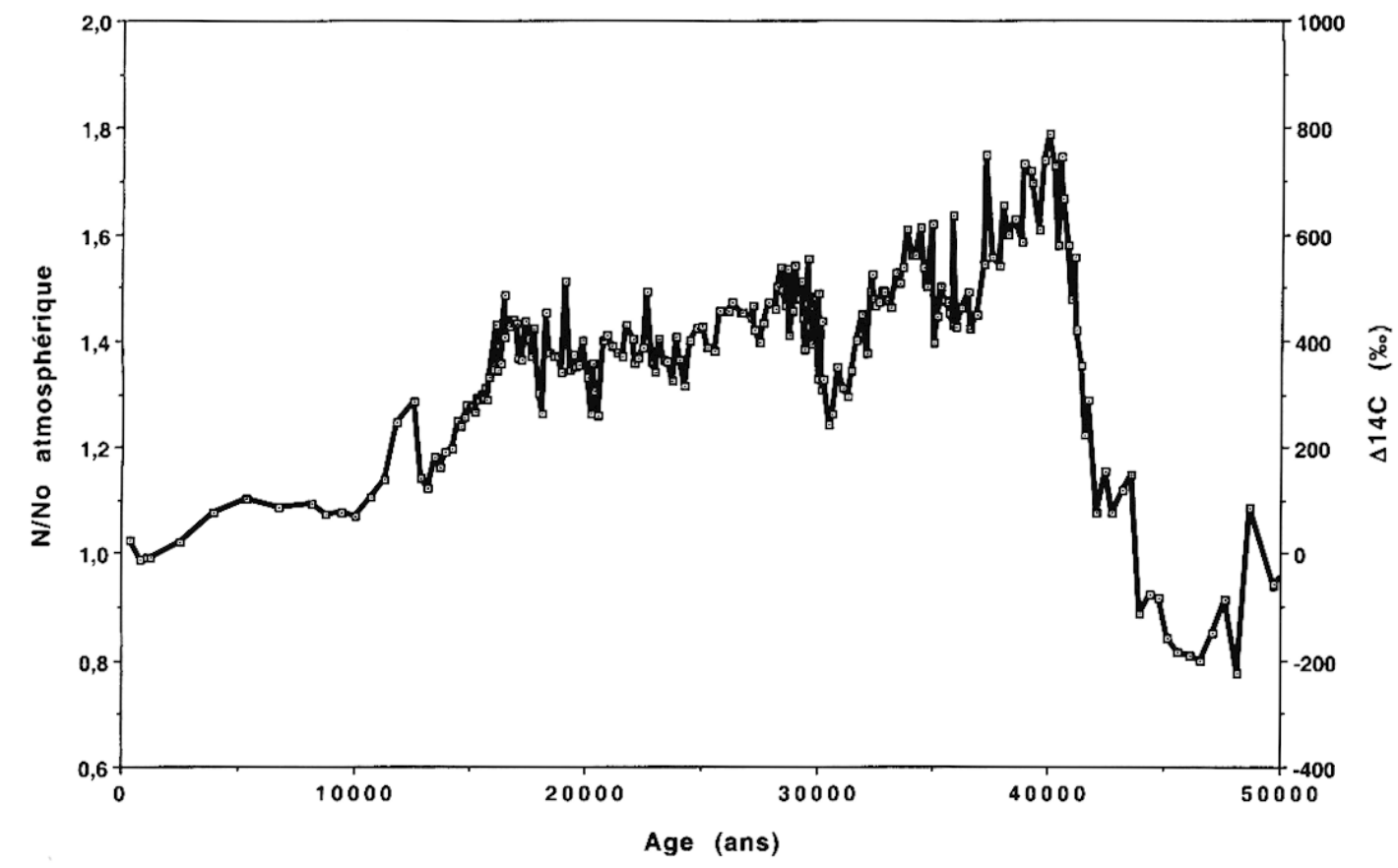

Fig. 1 : Variations de la teneur en carbone 14 de l'atmosphère exprimée en $\Delta^{14} \mathrm{C}$ et/ou par rapport la concentration (No) mesurée en 1950 d'après les données de Hughen et al. (2004). Jusqu'à 25.000 ans, un consensus existe pour considérer fiables ces données.

Fig. 1: Variations of the carbon-14 content in the atmosphere expressed in $\Delta^{14} \mathrm{C}$ and/or by ratio of the concentrations in the past ( $N$ ) versus concentration (No) measured in 1950; data from Hughen et al. (2004). A consensus exists to consider reliable these data for the last 25,000 years. 
du rapport ${ }^{14} \mathrm{C} /{ }^{12} \mathrm{C}$ observée depuis les 30.000 dernières années ne peut pas être expliquée par ces fluctuations. La comparaison des variations temporelles des cosmonucléides ${ }^{14} \mathrm{C},{ }^{10} \mathrm{Be}$ et ${ }^{36} \mathrm{Cl}$ observées aux différentes bandes de latitude fournit un argument majeur. Les enregistrements de ces paramètres démontrent que c'est principalement aux basses latitudes mais assez peu dans les zones polaires que se produit cette modulation à long terme. $\mathrm{Ce}$ serait donc le champ magnétique qui serait responsable, en fait le champ dipolaire de la Terre dont la composante horizontale est maximale au niveau de l'équateur.

Le plus important pour le contrôle de la production du ${ }^{14} \mathrm{C}$ est donc l'intensité du champ magnétique terrestre. En effet, celui-ci agit comme une sorte de blindage contre toute particule chargée électriquement (comme les protons cosmiques à l'origine de la production $d{ }^{14} \mathrm{C}$ ). Ainsi, plus l'intensité du champ magnétique est importante moins la quantité de protons cosmiques interagissant avec les hautes couches de l'atmosphère est grande et plus petite est la production de ${ }^{14} \mathrm{C}$.

Si la physique du phénomène était donc connue depuis longtemps, il restait cependant à savoir comment variait l'intensité du champ magnétique dans le passé. Bucha et collaborateurs entre 1967 et 1970 montrent une étroite corrélation entre la variation de l'intensité du champ magnétique et les variations à long terme du rapport ${ }^{14} \mathrm{C} /{ }^{12} \mathrm{C}$ dans l'atmosphère (Bucha et al., 1970). M. Barbetti, dans les années 1970-80, montrera, en mesurant les paléo-intensités du champ magnétique sur des coulées de laves volcaniques ou des échantillons archéologiques comme des terres cuites de foyers, le rôle prédominant du champ magnétique sur les variations de la production de carbone 14 (Barbetti, 1980).

Il faudra attendre le début des années 1990 pour disposer des mesures des variations relatives des paléo-intensités sur des carottes marines (Tric et al., 1992 ; Guyodo et Valet, 1996). Pour les 50.000 dernières années, les conséquences des variations d'intensité du champ magnétique terrestre sur la production ${ }^{\mathrm{de}}{ }^{14} \mathrm{C}$ ont ainsi pu être estimées : l'essentiel de la longue tendance au rajeunissement pour les périodes anciennes est en bon accord avec la diminution d'intensité du champ magnétique jusqu'à 25.000 ans (fig. 2).

Par ailleurs, la diminution du rapport ${ }^{14} \mathrm{C} /{ }^{12} \mathrm{C}$ depuis 35.000 ans comparée aux mesures de la teneur en ${ }^{10} \mathrm{Be}$ de sédiments marins (Frank et al., 1997) indique que la diminution du champ magnétique terrestre a favorisé conjointement la production des deux cosmonucleides ${ }^{14} \mathrm{C}$ et du ${ }^{10} \mathrm{Be}$. Plus récemment, les travaux de Wagner $e t$ al. (2000), avec un autre isotope cosmogénique $\left(\mathrm{le}^{36} \mathrm{Cl}\right)$ mesuré dans des carottes de glace groenlandaises confirment ces résultats : durant les deux excursions magnétiques de Mono Lake et de Laschamp respectivement datées de 32 ou $35 \mathrm{ka}$ et de 39 ou $41 \mathrm{ka}$ suivant les chronologies relatives des carottes de glace groenlandaise (GRIP ou GISP2) la production chlore 36 a pratiquement doublé. Les mêmes conséquences sont prévisibles pour le ${ }^{14} \mathrm{C}$ et en bon accord avec les observations de Volker $e t$ al., (1998) ou Laj et al., (2002) sur des carottes marines.

\section{- LES ÉCHANGES ENTRE RÉSERVOIRS}

Les carottages effectués dans les calottes de glaces polaires ont permis de mettre en évidence un autre phénomène. En analysant la composition chimique des bulles d'air emprisonnées dans la glace, les équipes de glaciologues de Berne et du Laboratoire de Glaciologie de Grenoble ont montré que la teneur en gaz carbonique de l'atmosphère avait varié au cours du temps (Barnola et al., 1987). Lors du dernier maximum glaciaire, elle se trouvait réduite d'environ $30 \%$ par rapport à celle de la période préindustrielle. Ceci signifie, en première approximation, qu'à champ magnétique et flux cosmique constants, le gaz carbonique de l'atmosphère est enrichi en ${ }^{14} \mathrm{C}$ puisque, comme nous l'avons vu précédemment, cet isotope est produit essentiellement à partir de l'azote, constituant majeur de l'air (78\%).

Cette variation de la teneur en $\mathrm{CO}_{2}$ (fig. 3) résulte des modifications des taux d'échange entre les différents réservoirs. Pendant les périodes glaciaires, la pénétration du $\mathrm{CO}_{2}$ (et avec lui le ${ }^{14} \mathrm{C}$ nouvellement produit) dans l'océan était réduite du fait de l'extension de la couverture de glace et du ralentissement de la circulation océanique profonde. Ce phénomène a entraîné une augmentation des teneurs en ${ }^{14} \mathrm{C}$ de l'atmosphère. De même,

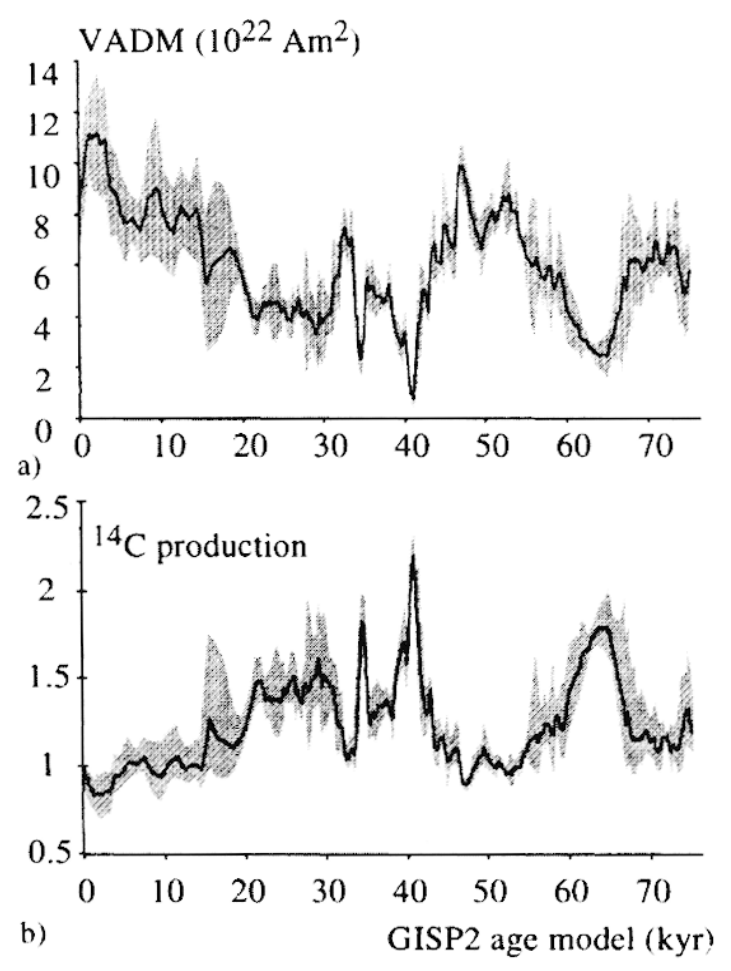

Fig. 2 : a) Variations de l'intensité du champ magnétique terrestre au cours des derniers $70 \mathrm{ka}$. b) Variations de la production de ${ }^{14} \mathrm{C}$ dans l'atmosphère calculées d'après les variations de l'intensité du champ magnétique terrestre au cours des derniers 70 ka (d'après Laj et al., 2002).

Fig. 2: a) Variations of the intensity of the terrestrial magnetic field during the last $70 \mathrm{ka}$. b) Variations of the production of ${ }^{14} \mathrm{C}$ in the atmosphere calculated using variations of the intensity of the terrestrial magnetic field during the last $70 \mathrm{ka}$ (from Laj et al.. 2002). 


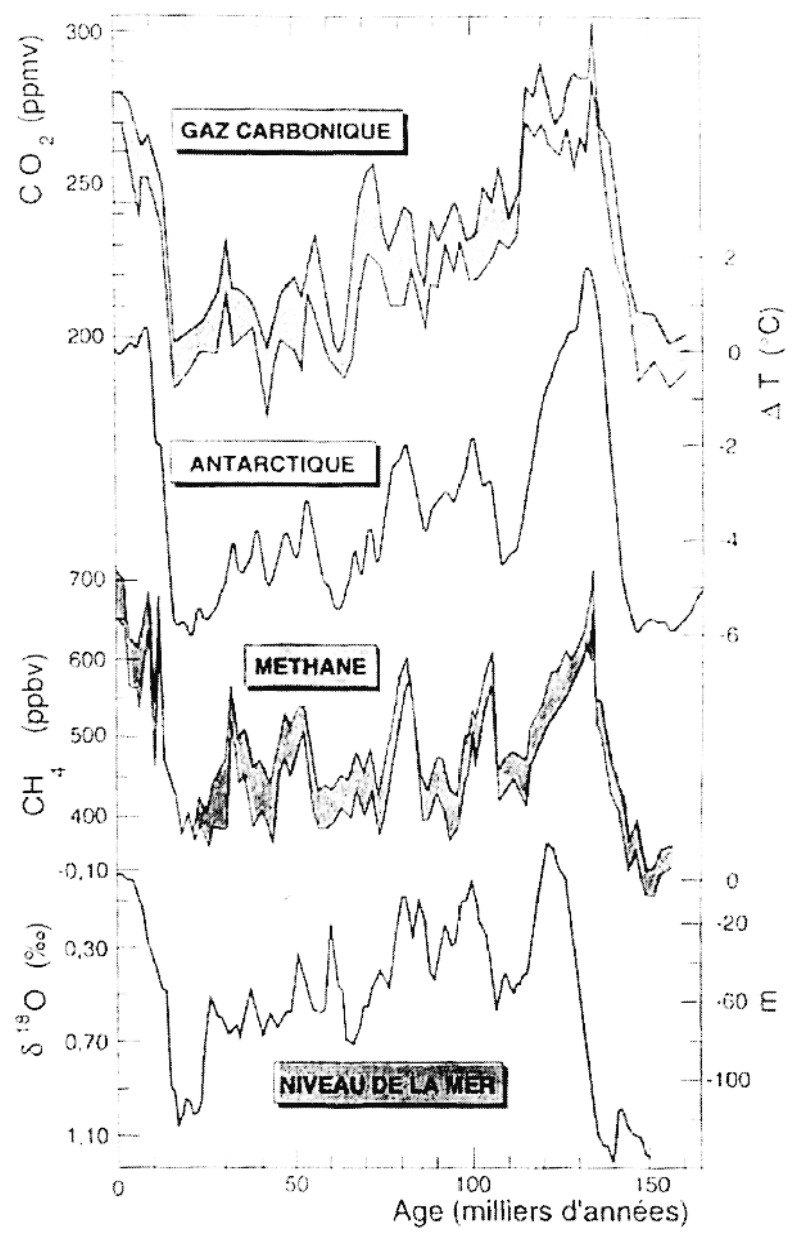

Fig. 3 : Variation des teneurs en $\mathrm{CO}_{2}$ et en méthane atmosphérique, de la température d'après les enregistrements de glace Vostok (Antarctique) et variation du niveau des mer durant les derniers 150 ka (d'après Besnard et al., 1992).

Fig. 3: Variation of the contents of $\mathrm{CO}_{2}$, atmospheric methane, temperatures recorded in ice Vostok ice core (Antarctica) and variation of the sea level during the last 150ka (from Besnard et al., 1992).

la biosphère continentale était aussi très réduite. Tous ces changements ont eu des répercussions sur le rapport atmosphérique ${ }^{14} \mathrm{C} /{ }^{12} \mathrm{C}$. Différentes estimations suggèrent une augmentation, de $10 \%$, au maximum selon Bard (1997). Ces effets de second ordre permettraient de mieux rendre compte des petites divergences observées. De plus, ils montrent que des contributions internes au cycle du carbone ont pu engendrer des écarts entre les âges ${ }^{14} \mathrm{C}$ bruts et les âges réels de plusieurs siècles qui se surimposent aux variations liées à des changements du taux de production par le rayonnement cosmique.

\section{LE CALIBRAGE DES ÂGES ${ }^{14} \mathrm{C}$}

A ce stade de la discussion, la méthode en terme de datation absolue par le ${ }^{14} \mathrm{C}$ est donc inapplicable, sauf $\mathrm{si}$ l'on est capable de se libérer de l'hypothèse de Libby sur la teneur initiale en ${ }^{14} \mathrm{C}$. Ainsi est née l'idée du calibrage des âges ${ }^{14} \mathrm{C}$, c'est-à-dire pouvoir reconstituer la variation rapport ${ }^{14} \mathrm{C} /{ }^{12} \mathrm{C}$ dans le passé et ainsi être en mesure de corriger les âges ${ }^{14} \mathrm{C}$.

La plupart des objets datés au ${ }^{14} \mathrm{C}$ ont extrait leur carbone de l'atmosphère, soit directement comme les plantes par la photosynthèse, soit indirectement comme les ani- maux via la chaîne alimentaire. Connaitre la teneur initiale en ${ }^{14} \mathrm{C}$ d'un échantillon revient donc à étudier l'évolution du rapport ${ }^{14} \mathrm{C} /{ }^{12} \mathrm{C}$ dans l'atmosphère. La comparaison entre un âge ${ }^{14} \mathrm{C}$ et un âge réel (abusivement appelé âge calendaire) d'un même fossile permet de reconstruire le rapport ${ }^{14} \mathrm{C} /{ }^{12} \mathrm{C}$ de l'époque.

Plusieurs techniques sont utilisées pour calibrer le radiocarbone et reconstituer les teneurs atmosphériques en ${ }^{14} \mathrm{C}$ au cours du temps.

\section{1 - La dendrochronologie}

En comptant les cernes de croissance des arbres à partir de l'actuel, on obtient l'âge de chacun d'eux et, en étudiant minutieusement la succession d'anneaux d'arbres vivants ou fossiles (dendrochronologie), on arrive à repérer des séquences caractéristiques permettant de faire des recoupements d'un tronc à un autre et d'obtenir une très longue série chronologique de bois d'âges connus. $\grave{A}$ partir de la mesure de l'activité ${ }^{14} \mathrm{C}$ de quelques cernes successifs, il devient possible de retrouver l'activité ${ }^{14} \mathrm{C}$ de l'atmosphère tout au long de cette série (fig. 4) (De Vries, 1958).

Malheureusement, il est extrêmement difficile d'étendre ce dendro-calibrage car les bois fossiles utilisables en dendrochronologie sont très rares au-delà de 11.000 ans BP. En effet, la période entre 11.000 et 70.000 ans BP correspond à la dernière glaciation et les forêts actuelles des latitudes tempérées étaient rares ou en grande partie recouvertes par des calottes glaciaires ou remplacées par des paysages de steppes et de toundras.

\section{2 - Les sédiments varvés}

Pour poursuivre le calibrage au-delà de 11.000 ans, des techniques de comptage similaires ont été utilisées : l'existence de sédiments varvés allait permettre d'aller un peu plus loin dans le passé. Il suffit, théoriquement, de compter ces varves pour obtenir l'échelle de temps " calendaire " et de mesurer l'activité ${ }^{14} \mathrm{C}$ correspondante de macro restes végétaux aériens contenus dans ces varves. Cette stratégie a été utilisée, avec succès, sur des enregistrements lacustres européens (notamment le Lac Gosciaz en Pologne, Goslar et al., 1995) ou sur les marges continentales (Bassin de Cariaco, au large du Vénézuela, Hughen et al., 2004) pour les périodes inférieures à 15.000 ans. Un seul enregistrement lacustre couvre les 45.000 dernières années ; il s'agit du lac Suigitsu au Japon pour lequel Kitagawa et Van der Plicht montrent de très forts rajeunissements des âges ${ }^{14} \mathrm{C}$ entre 30 et 40.000 ans (Kitagawa et Van der Plicht, 1998, 2000). Pour les époques les plus récentes, tous ces enregistrements montrent un accord excellent avec ceux obtenus avec la dendrochronologie. Au-delà, les comptages de varves, souvent incertains, n'offrent pas assez de garanties pour les utiliser en calibrage.

\section{3 - Les coraux}

Parallèlement, $d$ 'autres techniques ont été utilisées pour le calibrage : la datation des coraux des récifs tropicaux. En effet, ces coraux peuvent être datés à la fois par le ${ }^{14} \mathrm{C}$ et par la méthode des déséquilibres dans la famille 


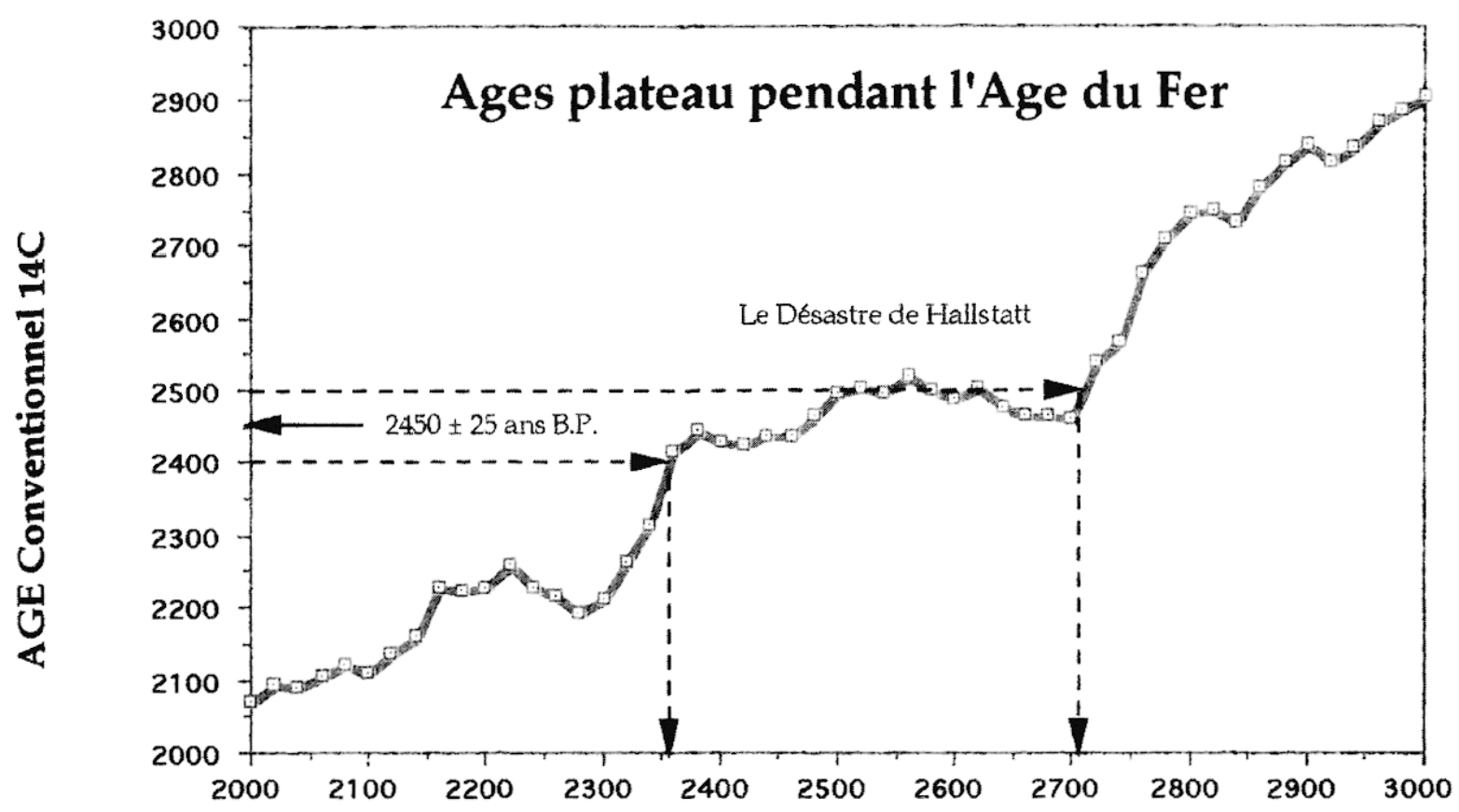

Années calendaires B.P.

Fig. 4 : Exemple de courbe de calibrage par la dendrochronologie. Exemple d'un âge plateau pendant l'âge du fer ancien européen (d'après Fontugne, 2002). L'intervalle de dates calibrées est donné au niveau de confiance $95,4 \%$ ( 2 sigma).

Fig. 4: Example of calibration curve by the dendrochronology with the "age plateau "during the of European old Iron Age (from Fontugne, 2002). The intervalle of calibrated dates is given at level of confidence $95,4 \%$ (2 sigma).

de l'uranium communément appelée "uranium-thorium", mais il faut utiliser des techniques récentes de spectrométrie de masse par accélérateur pour le ${ }^{14} \mathrm{C}$ ou par thermoionisation pour U/Th. Grâce à ces nouvelles techniques, il a été possible de reconstituer partiellement les fluctuations du rapport atmosphérique ${ }^{14} \mathrm{C} /{ }^{12} \mathrm{C}$ jusqu'à environ 45.000 ans B.P., limite pratique de l'utilisation du chronomètre radiocarbone. Les résultats obtenus notamment par E. Bard et al. (1998) sur les coraux des îles de La Barbade, Mururoa, Tahiti et Nouvelle-Guinée révèlent qu'entre 30.000 et 20.000 ans BP le rapport ${ }^{14} \mathrm{C} /{ }^{12} \mathrm{C}$ dépassait celui mesuré aujourd'hui de 40 à $50 \%$ et qu'il a diminué entre 18.000 et 30.000 ans B.P. (fig. 5). Néanmoins, plus que les incertitudes sur les corrections d'âge réservoir des dates radiocarbone, la difficulté de collecter des coraux de la dernière période glaciaire aujourd'hui sous 40 à $120 \mathrm{~m}$ d'eau, constitue la vraie limitation de cette approche.

\section{4 - Les stalagmites}

Récemment, la même technique couplant âges ${ }^{14} \mathrm{C}$ et âges U/Th a été utilisée sur des stalagmites provenant des Bahamas. Ainsi, Beck et al. (2001), après avoir évalué la proportion de carbone mort (dépourvu de ${ }^{14} \mathrm{C}$ ) qui provient de la formation géologique dans laquelle la grotte s'est formée, montrent que l'activité du ${ }^{14} \mathrm{C}$ atmosphérique présente de larges variations. Ces concrétions présentent aussi de forts rajeunissements des âges radiocarbone entre 30 et 40.000 ans. Ces résultats ne sont guère encourageants pour le calibrage, car les pics de production de ${ }^{14} \mathrm{C}$ enregistrés à cette époque se traduisent par des activités ${ }^{14} \mathrm{C}$ mesurées relativement constantes sur une longue durée (âge plateau). La difficulté de cette approche réside d'une part dans l'estimation de la proportion de carbone mort qui n'a aucune raison de rester constante dans le temps et d'autre part, d'évaluer le degré de fermeture du système chimique U/Th (départ ou ajout d'un de ces éléments) dans ces concrétions.

\section{5 - Corrélations avec des enregistrements datés}

La difficulté de trouver des enregistrements sédimentaires datables par deux méthodes radiométriques indépendantes a conduit à privilégier une autre approche. La datation absolue d'enregistrements sédimentaires marins est généralement effectuée par une corrélation étroite entre paramètres physiques ou isotopiques et les variations des paramètres isotopiques similaires datés par comptage de strates dans les carottes de glace du Groenland (forages GRIP et GISP). La datation ${ }^{14} \mathrm{C}$ de fossiles carbonatés (foraminifères) dans ces carottes de sédiments marins permet, après correction de l'âge réservoir océanique, d'évaluer le biais chronologique de la datation ${ }^{14} \mathrm{C}$ et ainsi reconstituer le rapport atmosphérique ${ }^{14} \mathrm{C} /{ }^{12} \mathrm{C}$. Les résultats de Volker et al. (1998) et Hughen et al. (2004) fournissent une illustration marquante des performances de cette approche. Néanmoins, des incertitudes subsistent car rien ne garantit que l'établissement de la corrélation entre les différents enregistrements soit parfait. Un taux de sédimentation constant, l'absence de petites lacunes de sédimentation dans les carottes sédimentaires ou dans les carottes de glaces du Groenland et la constance sur une si longue période des âges réservoir marins utilisés pour les corrections ne peuvent être a priori exclus. 


\section{QUELLE EST LA BONNE COURBE DE CALIBRAGE?}

L'ensemble de ces reconstitutions sont toutes en bon accord jusqu'à 20-25.000 ans, par construction pourrait-on dire puisque la validation de ces courbes de calibrage dépend en premier lieu de leur accord avec les courbes fournies par la dendrochronologie pour les périodes plus récentes que 15.000 ans. Au-delà de 25.000 ans, toutes les données ou courbes de calibrage proposées présentent de sérieuses divergences (fig. 5).

Pour illustrer les difficultés rencontrées pour seulement estimer des corrections d'âges ${ }^{14} \mathrm{C}$, on peut se reporter aux exemples suivants (fig. 6). Pour la période 30-40.000 ans, les corrections à appliquer aux âges ${ }^{14} \mathrm{C}$ pourraient varier entre plus de 5000 ans pendant les événements magnétiques à 1000 à 2500 ans en dehors de ceux-ci. $\mathrm{Si}$ les événements magnétiques débutent et finissent de manière abrupte, deux échantillons séparés réellement de quelques dizaines (centaines) d'années (l'un dans l'autre en dehors de l'anomalie magnétique) peuvent différer de plusieurs milliers d'années en âge ${ }^{14} \mathrm{C}$ B.P. C'est l'effet inverse de celui observé pour les périodes d'âge plateau. De ce point de vue, il faut donc rester très prudent car une date ${ }^{14} \mathrm{C}$ B.P. considérée comme trop jeune (et souvent rejetée par l'utilisateur) peut, en réalité, se révéler tout à fait exacte. D'une manière générale, pour cette période couvrant la transition entre Paléolithique moyen et supérieur, le traitement statistique de séries de dates ${ }^{14} \mathrm{C}$ B.P. en fréquence de distribution couvrant cette période est un exercice à haut risque, d'autant plus risqué que les incer-

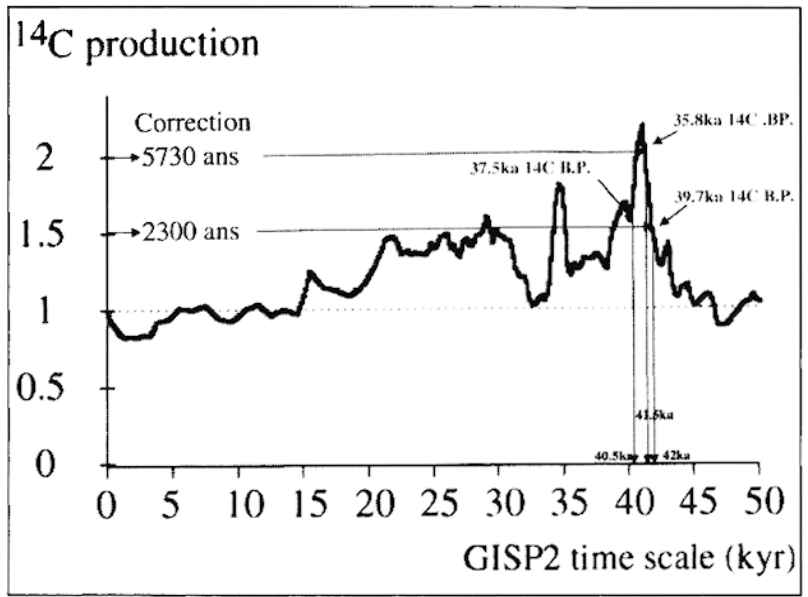

Fig. 6 : Variations de la production du carbone 14 résultant des variations de l'intensité du champ magnétique durant les $\mathbf{5 0 . 0 0 0}$ dernières annnées (d'après Laj et al., 2000, 2002). En ordonnée sont portées les variations relatives de l'intensité du champ magnétique et les corrections correspondantes à appliquer aux âges ${ }^{4} \mathrm{C}$. Exemple d'inversion possible pour des âges radiocarbone au voisinage dc ces pics de production.

Fig. 6: Variations of the production of carbon- 14 resulting from the variations of the intensity of the magnetic field during the last 50,000 years (from Laj et al., 2000, 2002). In ordinate are carried the variations relative of the intensity of the magnetic field and the corresponding corrections to apply to the ages ${ }^{14} \mathrm{C}$. Examples of possible age inversion for ages radiocarbon in the vicinity of these peaks of production.

titudes sur les âges sont souvent négligées (voir Djindjian, 1999 ; D’Errico et Sanchez Goñi, 2003). Cette nonlinéarité des âges ${ }^{14} \mathrm{C}$ B.P. entre 30 et 45.000 ans, implique que ce type de traitement statistique n'est rigoureux

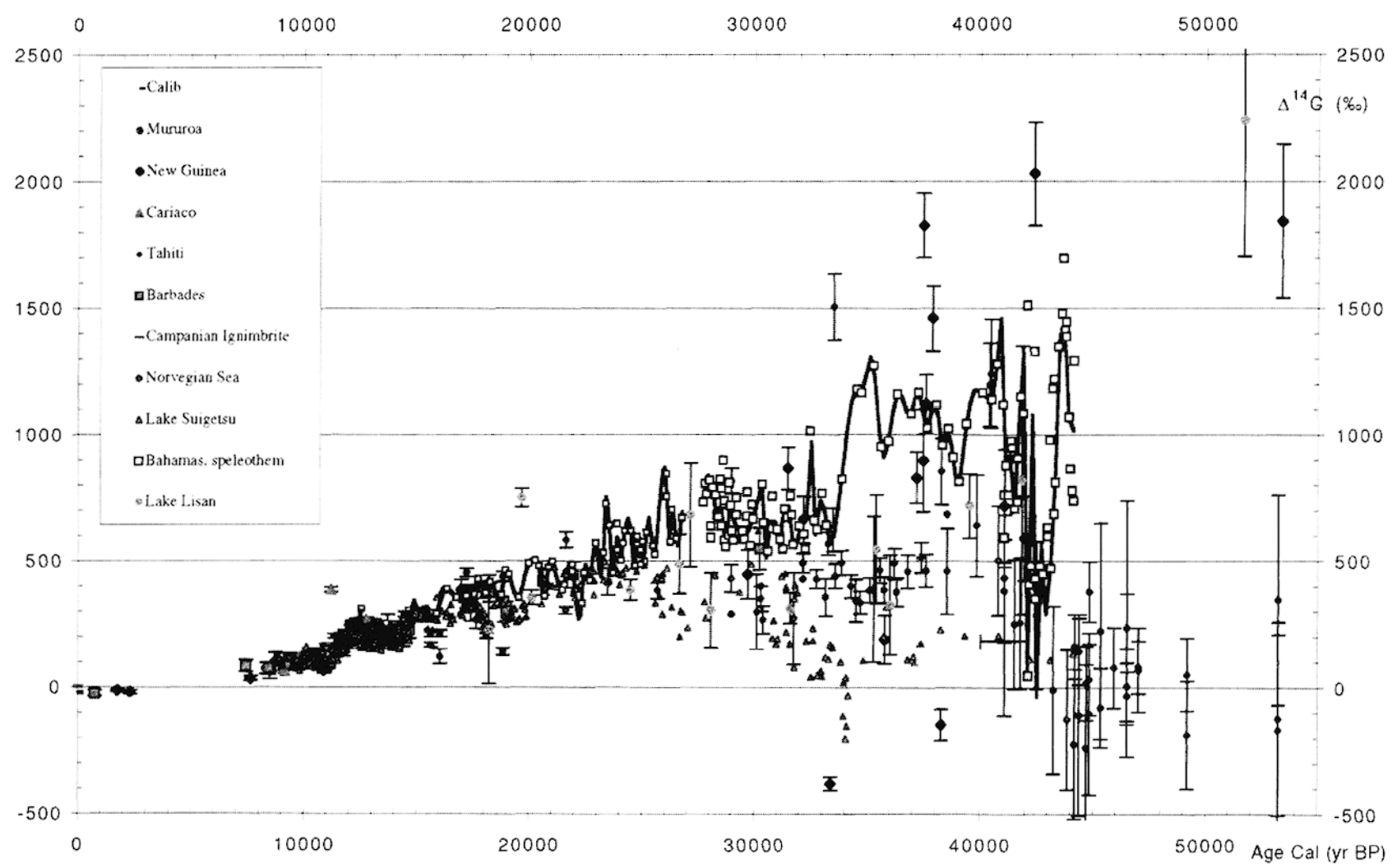

Fig. 5 : Compilation des différents enregistrements proposés pour le calibrage des âges ${ }^{14} \mathrm{C}$ (d'après $\mathrm{M}$. Paterne, non publié). La dispersion des valeurs de $\Delta^{14} \mathrm{C}$ au delà de $25 \mathrm{ka} \mathrm{pour} \mathrm{les} \mathrm{différents} \mathrm{enregistrements} \mathrm{est} \mathrm{mal} \mathrm{expliquée.}$

Fig. 5: Compilation of various records proposed for the calibration of the ages ${ }^{14} \mathrm{C}$ (from Mr. Paterne, unpublished). The dispersion of the values of $\Delta^{14} \mathrm{C}$ beyond $25 \mathrm{ka}$ for the various recordings is poorly understood. 
que sur les âges calibrés, hélas, indéterminables dans l'état actuel des différentes courbes de calibrage.

De même, selon le type de courbe de calibrage choisie, les écarts d'âge peuvent être importants. Ainsi, les données de Beck et al. (2001) ne sont pas en accord avec les données de coraux ou des varves du Lac Suigitsu ou celles de Cariaco publiées par Hughen et al. ou encore avec les sédiment de la marge ibérique de Bard et al. (2004b). À titre d'exemple (fig. 7), en considérant deux échantillons datés au ${ }^{14} \mathrm{C}$ de 31 et 32.000 ans, la correction donnerait respectivement 34 et 39.000 ans selon Voelker et al. (1998), 33 et 34.000 ans selon Kitagawa et Van der Plicht, 37 et 39.000 ans selon Beck et al. (2001) et 35.5 et 36.000 ans selon Bard et al. $(2004 \mathrm{a}, \mathrm{b})$ ou Hughen et al. (2004). Ce mode de correction effectuée de manière simpliste par projection orthogonale (interceptes) sur les courbes de corrections ne prend pas en compte l'erreur statistique sur la datation ${ }^{14} \mathrm{C}(1$ sigma $=200$ à 1000 ans). Il nous apparaît donc clairement, qu'en l'état actuel de connaissance sur les variations de la teneur en ${ }^{14} \mathrm{C}$ atmosphérique entre 25 et 45.000 ans, il n'est pas possible par la méthode $\mathrm{du}{ }^{14} \mathrm{C}$ de fournir une chronologie exploitable et a fortiori une chronologie absolue. De plus, toute corrélation temporelle établie pour cette période sur la seule base de datations radiocarbone offre une forte probabilité d'être incorrecte.

\section{CONCLUSIONS}

Depuis 30 ans, la datation par la méthode du carbone 14 a accompli des progrès considérables : en proposant d'une part, un diagnostic réaliste des difficultés et d'autre part, une thérapie basée sur l'utilisation des courbes de calibrage des âges radiocarbone. Jusqu'à 12.000 ans, ces courbes, essentiellement dendrochronologiques, sont continues, universelles et d'une grande fiabilité. Au-delà, jusqu'à 25.000 ans, les enregistrements sont plus discontinus, mais le calibrage reste encore très robuste. Entre 25 et 50.000 ans, en plus de la dégradation de la précision des mesures d'âge ${ }^{14} \mathrm{C}$, les divergences entre les courbes de calibrage sont notoires bien que chacune d'entre elles, considérée séparément, offre, a priori, toutes les garanties. Dans ces conditions, quelle courbe choisir? Il n'est pas possible de fournir aujourd'hui de réponse satisfaisante aussi faut-il demeurer extrêmement prudent dans l'utilisation des âges radiocarbone entre 25 et 50.000 ans. Faut-il produire de nouvelles courbes à partir d'enregistrements mieux contrôlés ? Certainement : le calibrage du radiocarbone nécessaire à la bonne utilisation de ce chronomètre est donc loin d'être définitif. Une description plus précise des variations du champ géomagnétique et du cycle du carbone autour de cette période 30-50.000 ans, pourra, à l'avenir, permettre de mieux comprendre les causes exactes des biais de cette méthode. Longtemps encore, l'irremplaçable datation carbone 14 pourra être considérée comme " la pire des méthodes à l'exclusion de toutes les autres".

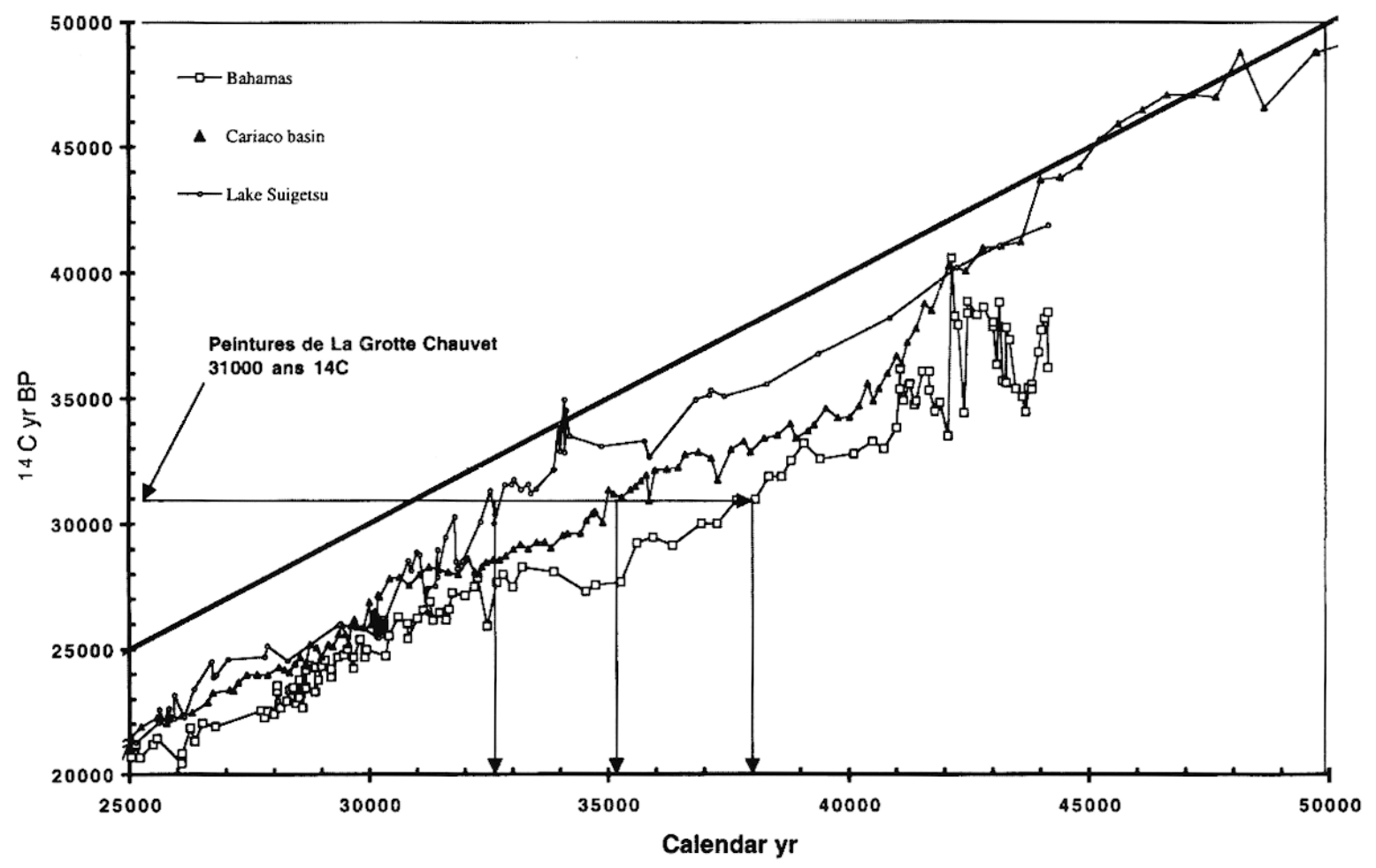

Fig. 7 : Les différentes possibilités de calibrage (cercles ouverts : Kitagawa et Van der Plicht, (2000), carrés ouverts : Beck et al., 2001, et triangles pleins : Hughen et al., 2004, la droite représente égalité des âges radiocarbone et réels) pour les âges du Paléolithique supérieur (d'après Bard et al., 2004a). Un constat de désaccord.

Fig. 7: Various possibilities of calibration (open circles: Kitagawa and Van der Plicht (2000), squares open: Beck and al., (200l), and full triangles: Hughen and al. (2004). the strait line represents equality between calendar and radiocarbon ages) for the ages of the Upper Paleolithic (from Bard et al., 2004a). A report of dissension. 


\section{REMERCIEMENTS}

Mes remerciements s'adressent particulièrement à Martine Paterne et Edouard Bard pour les nombreuses aides et suggestions qu'ils m'ont prodiguées.

\section{BIBLIOGRAPHIE}

BARBETTI, M., 1980 - Geomagnetic strength over the last 50000 years and changes in atmospheric ${ }^{14} \mathrm{C}$ concentrations. Radiocarbon, $22,192-199$

BARD, E., 1997 - Nuclide production by cosmic rays during the last ice age. Science, 277, 532-533.

BARD, E., ROSTEK, F. \& MÉNOT-COMBES, G., 2004a - A better radiocarbon clock. Science, 303, 178-179.

BARD, E., ROSTEK, F. \& MÉNOT-COMBES, G., 2004b - Radiocarbon calibration beyond $20,00014 \mathrm{C}$ yr B.P. by means of planktonic foraminifera of the Iberian Margin. Quaternary Research, 61, 204-214.

BARD, E., HAMELIN, B., FAIRBANKS, R.G. \& ZINDLER, A. 1990 - Calibration of ${ }^{14} \mathrm{C}$ timescale over the past 30,000 years using mass spectrometric U-Th ages from Barbados corals. Nature, 345, $405-410$.

BARD, E., ARNOLD, M., HAMELIN, B., TISNERAT-LABORDE, N. \& CABIOCH, G., 1998 - Radiocarbon calibration by means of mass spectrometry $230^{\mathrm{TI}} / 234 \mathrm{U}$ and $14 \mathrm{C}$ ages of corals. An updated data base including samples from Barbados Mururoa and Tahiti. Radiocarbon, 40, 1085-1092.

BARNOLA, J.M., RAYNAUD, D., KOROTKEVICH, Y.S. \& LORIUS, C., 1987 - Vostok ice core provides 160 000-year record of atmospheric $\mathrm{CO}_{2}$. Nature, 329, 408-414.

BECK, J.W., RICHARDS, D.A., EDWARDS, R.L., SILVERMAN, B.W., SMART, P.L., DONAHUE, D.J., HERRERA-OSTERHELD, S., BURR, G.S., CALSOYAS, L., JULL, A.J.T. \& BIDDULPH, D., 2001 - Extremely large variations of atmospheric ${ }^{14} \mathrm{C}$ concentration during the last glacial period. Science, 292, 2453-2458.

BESNARD, D., JOUZEL, J., LAJ, C. \& MELTZ, B., 1992 - Evolution du climat et de l'environnement global. In: Le livre bleu de l'environnement. Ed. CEA, 1-10.

BUCHA, J., TAYLOR, R.E. \& HARVEY, E.W., 1970 - Geomagnetic intensity: changes during the past 3000 years in the western hemisphere. Science, 168, 111-114.

DAMON, P. \& LINICK, T.W., 1986 - Geomagnetic-heliomagnetic modulation of atmospheric radiocarbon production. Radiocarbon, $28,266-278$

DÉLIBRIAS, G., 1985 - Le carbone 14. In : Méthodes de datation par les phenomènes nucléaires naturels : applications. Eds : Roth E. \& Poty B., Collection CEA, Masson (Paris), 421-458.

DE VRIES, H., 1958 - Variations in concentration of radiocarbon with time and location on earth. Proc. Kononkl. Ned Akad Wetenschap Ser., B61, 94-102.

DJINDJIAN, F., 1999 - Datations ${ }^{14} \mathrm{C}$ du Paléolithique supérieur européen : bilan et perspectives. Actes Colloque C14 archéologie, Mémoire S.P.F. XXVI, 171-179.

D'ERRICO, F. \& SANCHEZ GOÑI, M.F., 2003 - Neandertal extinction and the millenial scale climatic variability of OIS 3. Quaternary Science Reviews, 22, 769-788.
EVIN, J., 2002 - Le Radiocarbone. In : Géologie de la Préhistoire, Miskowski J.C. (Ed). GEOPRE, Perpignan, 1181-1197.

FONTUGNE, M., 2002 - La dérive des âges carbone 14. In : Géologie de la Préhistoire, Miskowski J.C. (Ed). GEOPRE, Perpignan, 1199-2005.

FRANK, M., SCHWARZ, B., BAUMANN, S., KUBIK, P.W, SUTER, M. \& MANGINI, A., 1997 - A 200 kyr record of cosmogenic radionuclide production rate and geomagnetic field intensity from ${ }^{10} \mathrm{Be}$ in globally stacked deep-sea sediments. Earth and Planetary Science Letters, 149, 121-130.

GOSLAR, T., ARNOLD, M., BARD, E., KUC, T., PAZDUR, M.F., RALSKA-JASIEWICZOWA, M., ROZANSKI, K., TISNERAT, N., WALANUS, A., WICIK, B. \& WIECKOWSKI, K., 1995 High concentration of atmospheric ${ }^{14} \mathrm{C}$ during the Younger Dryas cold episode, Nature, 377, 414-417.

GUYODO, Y. \& VALET, J.-P., 1996 - Relative variations in geomagnetic intensity from sedimentary records: the past 200,000 years. Earth and Planetary Science Letters, 143, 23-36.

HUGHEN, K., LEHMAN, S., SOUTHON, J., OVERPECK, J., MARCHAL, O., HERRING, C. \& TURNBULL, J., $2004-{ }^{14} \mathrm{C}$ activity and global carbon cycle changes over the past 50,000 years. Science, 303, 202-207.

KITAGAWA, H. \& VAN DER PLICHT, J., 1998 - Atmospheric radiocarbon calibration to $45,000 \mathrm{yr}$ BP: Late glacial fluctuations and cosmogenic isotope production. Science, 279, $1187-1190$.

KITAGAWA, H. \& VAN DER PLICHT, J., 2000 - Atmospheric radiocarbon calibration beyond $11,900 \mathrm{cal}$ BP from lake Suigitsu laminated sediments. Radiocarbon, 42, 369-380.

LAJ, C., KISSEL, C., MAZAUD, A., CHANNELL, J.E.T. \& BEER, J., 2000 - North atlantic palaeointensity stack since 75ka (NAPIS-75) and the duration of Laschamp event. Philosphic Translation Royal Society. London A 358, 1009-1025.

LAJ, C., KISSEL, C., MAZAUD, A., MICHEL, E., MUSCHELER, R. \& BEER, J., 2002 - Geomagnetic field intensity, North Atlantic Deep Water circulation and atmospheric $\Delta^{14} \mathrm{C}$ during the last $50 \mathrm{Kyr}$ Earth Planetary Science Letters, 200, 177-190.

LIBBY, W. F., 1952 - Radiocarbon dating. University of Chicago Press, $124 p$.

MANGINI, A., LOMITSCHKA, M., EICHSTÄDTER, R., FRANK, M., VOGLER, S., BONANI, G., HAJDAS, I. \& PÄTZOLD, J., 1998 - Coral provides way to age deep water. Nature, $392,347$.

STUIVER, M. \& QUAY, P.D., 1980 - Changes in atmospheric carbon-14 attributed to a variable sun, Science, 207, 11-19.

STUIVER, M. \& POLACH, H., 1977 - Discusion reporting of ${ }^{14} \mathrm{C}$ data. Radiocarbon, 19, 355-363.

TRIC, E.,VALET, J.P., TUCHOLKA, P., PATERNE, M., LABEYRIE, L., GUICHARD, F., TAUXE, L. \& FONTUGNE, M., 1992 Paleointensity of the geomagnetic field during the last 80 thousand years. Journal Geophysical Research, 97, B6, 9337-9351.

VOLKER, A.H.L., SARNTHEIN, M., GROOTES, P.M., ERLENKEUSER, H., LAJ, C., MAZAUD, A., NADEAU, M.-J. \& SCHLEICHER, M., 1998 - Correlation of marine ${ }^{14} \mathrm{C}$ ages from the nordic seas with the GISP2 isotope record: implication for ${ }^{14} \mathrm{C}$ calibration beyond 25ka BP. Radiocarbon, 40, 517-534.

WAGNER, M., BEER, J., LAJ, C., KISSEL, C., MAZARIK, J., MUSCHELER, R. \& SYNAL, H.A., 2000 - Chlorine 36 evidence for the Mono Lake event in the Summit GRIP ice core. Earth and Planetary Science Letter, 181, 1-6. 